\title{
Yod
}

Revue des études hébraïques et juives

19 | 2014

Aharon Appelfeld, cinquante ans d'écriture

\section{Littérature et génocide : l'écriture testimoniale des enfants}

Literature and Genocide: Children's Testimonial Writing

שואה וספרות :כתיבה מתעדת של ילדים

\section{Frosa Pejoska-Bouchereau}

\section{OpenEdition}

Journals

Édition électronique

URL : https://journals.openedition.org/yod/1965

DOI : $10.4000 /$ yod. 1965

ISSN : 2261-0200

Éditeur

INALCO

Édition imprimée

Date de publication : 30 mai 2014

ISBN : 978-2-85831-214-6

ISSN : 0338-9316

Référence électronique

Frosa Pejoska-Bouchereau, «Littérature et génocide : l'écriture testimoniale des enfants 》, Yod [En ligne], 19 | 2014, mis en ligne le 16 avril 2014, consulté le 08 juillet 2021. URL : http://

journals.openedition.org/yod/1965; DOI : https://doi.org/10.4000/yod.1965

Ce document a été généré automatiquement le 8 juillet 2021.

\section{(c) (1) 8)}

Yod est mis à disposition selon les termes de la Licence Creative Commons Attribution - Pas d'Utilisation Commerciale 4.0 International. 


\title{
Littérature et génocide : l'écriture testimoniale des enfants
}

\author{
Literature and Genocide: Children's Testimonial Writing \\ שואה וספרות :כתיבה מתעדת של ילדים
}

Frosa Pejoska-Bouchereau

Les enfants survivants ne sauraient se remémorer

l'Holocauste à la manière des adultes. Leur contribution est inséparable de leur expérience vécue. Mais cette expérience, quoique limitée, est profonde. Rien d'étonnant à ce que la littérature de l'Holocauste soit née avec eux. (Aharon Appelfeld,

«L'Holocauste lorsqu'on est enfant », Le Nouvel Observateur no 2097 du 13 au 19 janvier 2005).

1 Comme aucun survivant avant lui et bien qu'il affirme éviter les abstractions ${ }^{1}$, Aharon Appelfeld théorise dans L'héritage nu une distinction entre les témoignages des adultes et les témoignages des enfants sur le génocide. Si pour les adultes les témoignages doivent être factuels, chronologiques et fidèles, pour les enfants - que l'on a refusé de considérer comme des témoins - faute de pouvoir reconstituer le passé par la mémoire, il s'agit de recourir à la fiction, à l'expression des sensations et des sentiments, en d'autres mots à la perception, pour procéder à une "reconstruction". Ce recours singulier à l'imaginaire et aux sens serait, selon Appelfeld, à l'origine de la naissance de la littérature de la Shoah. Notre questionnement aura pour sujet la définition du " témoignage » et sa place dans la littérature.

\section{Les témoins survivants}

2 Pour le survivant adulte, la mémoire chronologique (les noms, les lieux, les dates) est un point d'ancrage duquel il ne veut pas se départir. Une mémoire qui couplerait réalité et fiction serait d'emblée condamnée : « Toute évocation fictionnelle de l'Holocauste a 
été et est encore considérée comme un acte indigne de la gravité du sujet $»^{2}$. La fidélité à la réalité vécue est essentielle pour le survivant. Le jeu avec les mots et les formes, le recours à la création sont interdits. Seule est autorisée l'évocation des faits ${ }^{3}$. Aussi la plupart des écrits consacrés à la Shoah relèvent-ils d'un travail historique.

Or, les survivants s'éteignent et avec eux la caution d'authenticité. Pour qui écrit sur le sujet, qu'il soit historien ou écrivain, les survivants étaient et demeurent la « hantise ». Sans le survivant se pose la question de la préservation de la « dimension individuelle et intime que conférait le survivant à cette terrible expérience $»^{4}$.

Reste un autre type de témoin survivant : tous ceux qui étaient enfants au moment de l'événement. Toutefois, les enfants n'étaient pas comptabilisés parmi les survivants et leur mémoire n'était pas considérée comme telle, bien qu'un million et demi d'enfants soient morts dans la Shoah, soit $90 \%$ des enfants juifs d'Europe. Pour les survivants adultes l'enfant ne se souvient pas et «celui qui ne se souvient pas, c'est comme s'il n'avait jamais été là-bas $»^{5}$. Pourtant, immédiatement après la guerre, entre 1946 et 1949, plusieurs témoignages d'enfants furent publiés, en particulier dans les pays de l'Est.

Le nouveau comité ne connut pas de repos. Les anciens guettaient le moindre faux pas, et à chaque assemblée ils déposaient des contre-propositions, le mettaient face à ses erreurs, arguaient du fait que ses membres n'avaient pas connu la Shoah puisqu'ils étaient des enfants, et que les enfants ne se souviennent pas, et celui qui ne s'en souvient pas, c'est comme s'il n'avait jamais été là-bas. Le nouveau comité était prêt à rendre les clés, mais à qui ${ }^{6}$ ?

Difficile, par conséquent, pour l'enfant témoin de marquer sa présence là-bas en l'exprimant ici et maintenant, son propos étant d'emblée condamné à n'être pas légitime.

\section{La mémoire}

L'adulte témoigne en relatant et en révélant, mais aussi en dissimulant, car il lui est impossible de tout dire. Ce qu'il a vécu est si incroyable, pour lui-même et bien plus pour tous ceux qui ne l'ont pas vécu, qu'il se heurte à l'impossibilité de le comprendre. À cela s'ajoute qu'il lui faudrait admettre qu'il n'est plus tout à fait le même, avouer sa propre transformation. Les témoignages des adultes doivent être abordés à la fois pour ce qu'ils nous livrent et surtout, dit Appelfeld, pour ce qui y manque, le non-dit, l'indicible. En témoignant, ils pensent se soulager d'un fardeau, or ils comprennent vite qu'ils ont gardé le plus lourd pour eux.

7 Pour l'enfant, la mémoire fonctionne tout autrement. L'enfant est dans l'incapacité d'assimiler toute l'horreur de cette réalité, il ne peut assimiler que la "portion accessible $»^{7}$ à son esprit d'enfant. Contrairement à l'adulte, il ne peut convoquer le passé pour comparer parce que son enfance et sa jeunesse sont représentées par l'Holocauste qui était « comme dit le poète, le lait noir qu'ils tétaient matin, midi et soir $"^{8}$. Les enfants n'ont donc pas de passé ou plus précisément, n'ont pour seul passé que l'Holocauste. S'ils ont connu le bonheur auprès de leurs parents, il a été supplanté par la terreur et l'horreur; il a été effacé par le malheur. Démunis devant l'atrocité des événements, ils sont symboliquement " handicapés " ${ }^{9}$. Appelfeld dira : «Les années de guerre puis de vagabondage en Europe furent des années aveugles pour les enfants ${ }^{10}$." Pourtant, ces enfants n'ont fait qu'observer, contempler, mais d'un regard nu, 
apathique. Un regard qui a tout enregistré comme l'objectif d'une caméra dont ils se repasseront le film, inlassablement, pour y trouver un sens, une fois adulte, une fois doués de la maturité réflexive.

8 L'adulte, lorsqu'il raconte, exprime les sensations, les sentiments en termes généraux sans introspection avec une objectivité revendiquée et un point de vue collectif, un point de vue "extérieur». L'adulte essaie d'oublier et de s'oublier pour pouvoir se réinsérer dans le tissu social. L'enfant, quant à lui, est incapable de parler du génocide en termes historiques, théologiques ou moraux, la puissance de son témoignage réside dans un horizon plus limité : «Ils ne pouvaient parler que des peurs, de la faim, des couleurs, des caves, des gens qui étaient gentils avec eux ou de ceux qui les maltraitaient ${ }^{11}$.» Les témoignages des enfants sont l'expression de la souffrance individuelle d'une enfance meurtrie. Ils sont le point de vue "intérieur». Par conséquent, le témoignage des enfants revêt une forme littéraire.

Pour Appelfeld, il ne faut pas confondre «mémoires ", récits de vie que nous avons en abondance, et «écrits littéraires ». Dans ses mémoires, le survivant donne l'impression que tout s'est passé à l'extérieur de lui-même et que «la comptabilité spirituelle, si pareille chose existe, a essentiellement dressé le bilan de la société et n'a pas pris le monde de l'âme en considération ${ }^{12}$. Nathalie Zajde, dans son étude sur les enfants cachés, définit la frayeur comme une émotion extrême qui fait l'expérience de «la perte de son âme »; l'âme a "sursauté ", elle a été « raptée », " ravie », " capturée ", d'où l'impossible restitution de l'âme ${ }^{13}$. C'est, par conséquent, de cette perte qu'il importe de parler.

10 Les témoignages des enfants ont été rejetés par les adultes. Rejet d'autant plus fort qu'il a fallu s'opposer au négationnisme. Ce fut le cas du premier livre d'Appelfeld Fumée. Les éditeurs et les survivants y trouvèrent des défauts : on ne doit pas écrire de fiction sur la Shoah, il ne faut pas écrire sur la faiblesse des victimes, mais sur les actes héroïques, les héros, les révoltes. La réception, bien que favorable, classait l'œuvre «sur les marges de la Shoah $»^{14}$.

\section{Enfant caché}

11 Aharon Appelfeld a sept ans au début de la guerre, une guerre qui durera six longues années, pour ainsi dire autant, voire plus, que son enfance : «Ce furent des années où chaque minute, chaque seconde, chaque fraction de seconde était chargée au-delà de ce qu'elle était capable de contenir $»^{15}$. Enfant unique, vivant le bonheur auprès de ses parents, il voit subitement sa vie basculer dans un ordre temporel inconnu - « il n'y eut plus d'été ni d'hiver $»^{16}$ - où tous les repères avaient disparu, mettant le monde sens dessus dessous :

Dans le ghetto, les enfants et les fous étaient amis. Tous les repères s'étaient effondrés: plus d'école, plus de devoirs, plus de lever le matin ni d'extinction des feux la nuit. Nous jouions dans les cours, sur les trottoirs, dans des terrains vagues et de multiples endroits obscurs. Les fous se joignaient parfois à nos jeux. Eux aussi avaient tiré profit du chaos ${ }^{17}$.

12 Comme beaucoup d'enfants, il devient un enfant caché ${ }^{18}$ et assiste à sa métamorphose en un petit animal qui possède plusieurs terriers ${ }^{19}$, s'éloignant à ce point de sa vie antérieure qu'elle lui semble n'avoir jamais existé20. 

une répons les enfants et les adultes est inédit. Face à l'incroyable, difficile de trouver interdit tout questionnement. «Pendant la guerre, on ne tenait pas compte des enfants. Ils étaient le brin de paille que tout le monde piétinait ${ }^{21}$. Les enfants démunis devant une réalité qui les oublie et les nie ne savent pas vers qui se tourner pour avoir une réponse à leurs interrogations. Leurs questions, auxquelles ne peuvent répondre les adultes, suscitent la colère de ces derniers, aussi les enfants ne peuvent-ils plus questionner ${ }^{22}$. Ce questionnement se retrouvera dans leur écriture, car ils sont toujours en attente de la réponse qu'ils n'ont jamais obtenue.

\section{La contemplation}

14 Contraints au silence, ils vont s'adonner à la contemplation qui est une forme d'absence. La contemplation place l'individu en dehors de soi et des autres. Contempler c'est se tenir à distance, "à l'extérieur, légèrement surélevé et éloigné ${ }^{23}$; c'est sortir de soi, de son malheur et donc "diminuer la douleur», dira Appelfeld ${ }^{24}$. C'est aussi se faire oublier, se mettre à l'abri, se cacher. Par la contemplation, l'individu est spectateur des événements qui, bien qu'il les vive, lui paraissent étrangers. De même, il est spectateur des actes de ses semblables, car la " contemplation n'est pas une affaire entre soi et soi, elle concerne les gens, et peut-être les blesse-t-elle ». Appelfeld relate la réaction d'un vieil homme qui, un jour, lors d'une de ces contemplations, le gifla par deux fois en lui criant : "Maintenant tu ne regarderas plus. Maintenant tu sauras qu'on ne regarde pas. » Ces gifles sont qualifiées de " prémices de [s]a conscience ». Appelfeld prend conscience de son "aspiration à la clandestinité ", d'un sentiment de transgression. Il a l'impression d'avoir été pris en flagrant délit. Aussi, perdit-il la contemplation «spontanée et empathique » à laquelle il s'adonnait des heures durant pour une contemplation "furtive et volée » doublée d'une "nouvelle ruse » : épier les bruits. La contemplation remplit Appelfeld de « couleurs, sons, rythmes ${ }^{25}$, elle devient un réservoir de sensations dans lequel il puisera lorsqu'il commencera à écrire.

Si les humains, plus précisément les adultes, sont sans mots face à l'incroyable réalité, « la supériorité de la contemplation tient au fait qu'elle est dénuée de mots ${ }^{26}$, qu'elle peut se passer de ces mots, car "le silence des objets, des paysages vient à vous sans rien imposer $»^{27}$. Ce silence remplit l'enfant de sensations qui imprègnent son corps et sa mémoire.

16 Mais pourquoi les gifles ? Que ne doit-on pas regarder ? Les êtres se transforment à vue d'œil! Aussi voit-on ce que l'on ne doit pas voir. Eux aussi ne souhaitent pas être pris en flagrant délit. Par la contemplation, on devient témoin et juge de ce que l'on voit. Or, les gens ne sont plus ce qu'ils étaient. Ils ont été contraints de devenir autres, laids, physiquement et moralement, et cruels pour survivre. Les enfants dont on avait oublié la présence, voire l'existence, fonctionnent comme un miroir négatif dans lequel les humains voient leur nouveau visage et qu'ils tentent de briser par la violence des gifles répétées et de l'impérative interdiction verbale. Mais la gifle est aussi symbole du retour du devoir d'éducation, de formation des adultes vis-à-vis des enfants. Devoir qui avait été oublié. La gifle est le rappel du lien entre les générations qui avait été rompu. Ainsi, aussi paradoxal que cela puisse paraître, la contemplation, du fait même de la distance qu'elle instaure, d'un état de le hors de soi et hors des autres, ramène 
l'attention sur soi et les autres et recrée le lien. La contemplation est le lien entre le dehors et le dedans, nécessaire pour sortir les enfants de l'isolement dont ils souffrent.

Le génocide fait de ces enfants des orphelins. Avec la contemplation, ils redeviennent des fils. Tout comme le sommeil, la contemplation, par les visions qu'elle provoque, place les enfants en dehors de l'espace et du temps et leur permet de renouer les liens rompus avec les proches, en particulier les parents, et la vie antérieure.

Je restais des heures assis dans la forêt, à contempler la flore, ou près d'un ruisseau à suivre des yeux son cours. La contemplation me faisait oublier la faim et la peur, et des visions de la maison me revenaient. Ces heures étaient peut-être les plus plaisantes, s'il est permis d'utiliser cet adjectif lorsque l'on évoque le temps de la guerre. L'enfant sur le point d'être oublié dans cette solitude sauvage, ou d'être tué, redevenait le fils de son père et de sa mère, se promenant avec eux l'été dans les rues, un cornet de glace à la main, ou nageant avec eux dans le Prut. Ces heures de grâce me protégèrent de l'anéantissement spirituel. Plus tard aussi, après la guerre et la Aliyat Hanoar, sur les routes, je m'asseyais et contemplais, m'entourant de visions et de sons, me reliant à ma vie antérieure, heureux de ne pas être un parmi des milliers, privé de visage ${ }^{28}$.

Plus tard, Appelfeld écrivain utilisera précisément la contemplation comme procédé de distanciation à la base de son écriture romanesque: "Je ne savais pas alors que la contemplation me préparait en secret au rôle que le destin m'avait choisi ${ }^{29}{ }^{\text {}}$.

\section{Le corps-mémoire}

À la différence des adultes, pour les enfants, le corps plus que la mémoire se souvient. Les souvenirs sont imprimés dans les cellules du corps. Le corps-mémoire est cependant difficile à sonder. Chaque fibre du corps renferme des souvenirs qui émergent par fragments.

La mémoire, elle, n'est que brouillard épais, tunnel noir, réalité trouble enfouie à jamais. Quelques souvenirs en lambeaux qui apparaissent de manière fulgurante et disparaissent tout aussitôt. Comme effrayés eux-mêmes par ce qu'ils révèlent. Les souvenirs de la guerre ont la couleur du deuil ${ }^{30}$.

Chaque fois qu'il pleut, qu'il fait froid ou que souffle un vent violent, je suis de nouveau dans le ghetto, dans le camp, ou dans les forêts qui m'ont abrité longtemps. La mémoire, s'avère-t-il, a des racines profondément ancrées dans le corps $^{31}$.

21 Lorsqu'il s'agira d'écrire, Appelfeld interrogera son corps-mémoire. Une écoute attentive le replongera dans son passé lui permettant de rédiger ses premiers chapitres. Toutefois, même s'il n'invente pas, l'écriture est entièrement tournée vers l'expression des sens et non la description des faits. Or, « les gens réclamaient des faits, des faits précis, comme si en eux résidait le pouvoir de résoudre toutes les énigmes ${ }^{32}$ ». L'enfant ne peut relater des faits. Un fait est narré en fonction de son importance par rapport à un passé, mais aussi à un futur. Mais pour l'enfant tout est nouveau. Il lui est impossible, au moment où se produisent les faits, de sélectionner et de mémoriser l'essentiel, car il ignore dans le non-sens ce qui a de l'importance. Aussi enregistre-t-il la totalité, mais ne comprend que ce qui est accessible à sa sensibilité d'enfant. Sa mémoire est une pellicule muette qui nécessite un décryptage, un montage et une écriture ; une pellicule filmée en aveugle. «La guerre s'était terrée dans mon corps, pas 
dans ma mémoire. Je n'inventais pas, je faisais surgir des profondeurs de mon corps des sensations et des pensées absorbées en aveugle ${ }^{33}$.»

\section{L'impossible transmission mémorielle aux enfants} derniers étaient concernés et intéressés. La transmission se faisait par l'école ou les institutions, de façon "générale ou effrayante : avec des films sur Auschwitz " ${ }^{34}$ ou encore par des questions culpabilisantes posées aux rescapés sur leur manque de courage et de révolte, faisant d'eux des accusés qui manquaient d'arguments pour leur défense. De plus, le monde extérieur s'accommodait bien du silence, du refoulement et de l'oubli, aussi exigeait-il d'eux "qu'ils se renient et renient les souvenirs qu'ils avaient emportés $»^{35}$. Ce silence, qui, une fois encore, interdit le questionnement, irrite les enfants qui éprouvent le sentiment d'avoir été abusés sur leur généalogie, d'avoir été séparés de leur parenté, privés du passé : « Ils étaient irrités par ces années où leurs parents leur avaient dissimulé leurs vies antérieures, les avaient détachés de leurs grands-parents et de la langue de leurs grands-parents pour créer autour d'eux un monde fabriqué de toutes pièces, comme si rien ne s'était passé. $\rrbracket^{36}$ Quant à ceux qui parfois racontaient, ils ne pouvaient s'empêcher d'ajouter que les enfants n'étaient pas en mesure de comprendre. Dans la très belle œuvre de Carl Friedman, Mon père couleur de nuit ${ }^{37}$, nous mesurons combien il est difficile, voire impossible, de comprendre celui qui « a le camp », qui est possédé par le camp, car les enfants, s'ils ont eu la varicelle et la rubéole, n'ont pas eu le camp. Le père qui a eu et a toujours le camp veut comprendre lui aussi, mais il comprend encore moins maintenant qu'avant ${ }^{38}$. Est-il possible de raconter, de transmettre ce que l'on ne comprend pas?

Les enfants cachés ne racontent pas davantage à leurs enfants :

Les enfants de ma génération, dit Appelfeld, ont très peu parlé à leurs enfants de leur maison, et de ce qui leur était advenu pendant la guerre. L'histoire de leur vie leur a été arrachée sans cicatriser. Ils n'ont pas su ouvrir la porte qui menait à la part obscure de leur vie, et c'est ainsi que la barrière entre eux et leurs descendants s'est érigée. Il est vrai que, ces dernières années, ceux de ma génération tentent d'ébranler la muraille qu'ils ont construite de leurs propres mains, mais c'est un ébranlement infime, la barrière est épaisse et fortifiée, il est douteux qu'elle puisse être ébranlée ${ }^{39}$.

Nathalie Zajde ${ }^{40}$ dira que les enfants sont restés cachés dans le mutisme, se sentant honteux et responsables de ce qu'ils avaient vécu. S'ajoute à cela un sentiment d'incrédulité tant ce qu'ils racontent semble tout à la fois incroyable et impersonnel. Une disjonction se produit entre un récit qu'Appelfeld qualifie de plat, chronologique et extérieur qui ne révèle rien et une réalité vécue "extra-ordinaire ». Les mots qui se dévident au fil de la plume paraissent une pure fiction aux enfants eux-mêmes, la distance temporelle accentuant cet effet ${ }^{41}$.

\section{Le nécessaire oubli}

L'héritage paraissait nu, aussi nu que leur regard durant la guerre. En effet, les mots qui avaient fait défaut continuaient à trahir par leur impuissance à rendre la réalité des événements vécus. Un sentiment de doute, de soupçon, intrinsèque à l'emploi 
déroutant de mots inadéquats, habita les rescapés qui acceptèrent l'idée répandue qu'il était impossible d'écrire sur la Shoah ${ }^{42}$. Aussi, le premier texte sur la Shoah sera-t-il écrit dans un style documentaire, journalistique concernant le collectif, le général, avec une finalité sociale ${ }^{43}$.

Durant la guerre les enfants furent réduits au silence. Après la guerre, leurs témoignages écrits passèrent inaperçus. À l'instar des adultes, il restait aux enfants survivants un travail à accomplir : détruire la mémoire considérée comme un ennemi. Non seulement les enfants représentaient pour les adultes l'avenir, d'où la nécessité d'oublier le passé, mais ils avaient aussi cette particularité que pour leur corpsmémoire, où s'était terrée la guerre, il n'existait pas de mots pouvant exprimer la singularité de leur perception des événements. En conséquence, dissimuler, réprimer, voire supprimer les souvenirs était le travail de la génération d'Appelfeld. Après le génocide, il s'agissait donc de réduire à son tour la mémoire au silence. En lieu et place de la mémoire s'installait le nécessaire oubli et le mutisme, sorte de sommeil amnésique, faisant des rescapés des amputés ${ }^{44}$, tel le héros manchot dans Et la fureur ne s'est pas encore tue, ou pis encore, des morts ${ }^{45}$.

\section{La nouvelle forme}

Comment pour les enfants inscrire un sens quand les adultes survivants eux-mêmes ne pouvaient le faire? De plus, devaient-ils laisser l'oubli «creuser alors ses caves profondes $»^{46}$ et s'ancrer solidement en eux? N'étant pas considérés comme des témoins, ne pouvant pas relater des faits, ne pouvant pas utiliser le langage extérieur du général, mais ne pouvant pas non plus écrire sur leur expérience, exprimer leurs sentiments profonds inscrits dans les cellules de leur corps de peur d'être qualifiés d'égoïstes et de vulgaires, il leur restait le silence, mais ce silence semblait annuler ce qu'ils avaient vécu et par conséquent les annuler eux-mêmes. Face à ce dilemme, «la mémoire et l'oubli, la sensation d'être désarmé et démuni, d'une part, et l'aspiration à une vie ayant un sens, d'autre part $\aleph^{47}$, et en proie à la désespérance, les enfants chercheront de nombreuses années durant le moyen de « sortir leurs vies torturées des recoins où elles se cachaient $»^{48}$.

Quant à Appelfeld, il chercha également une «issue " à son besoin de commencer à converser avec lui-même, à trouver des mots pour habiller sa mémoire ${ }^{49}$. Ses premières tentatives d'écriture furent des échecs. Des poèmes et une chronique, qui décrivaient fidèlement ce qui lui était arrivé, n'étaient « rien de plus que des hurlements étouffés, des appels au secours, des cris adressés à Dieu. Dans leur désolation, ces poèmes étaient le gémissement d'un animal égaré, mais, au-delà de ça, il n'y avait rien ${ }^{50}$. Outre cette sensiblerie ${ }^{51}$ insupportable, ses souvenirs, bien que fidèles et vrais, lui apparaissaient peu fiables et fictionnels: "C'était l'histoire vraie de mon enfance, mais ce qui ressortait de mes pages avait une allure bizarre, peu convaincante et, qui pis est,

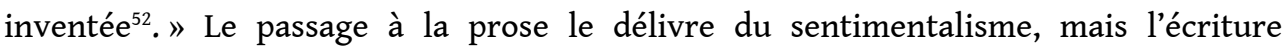
positiviste pratiquée l'éloigne de lui, le coupe de son passé en l'enracinant dans la nouvelle réalité israélienne, faisant de lui un homme nouveau issu de nulle part et de personne. Que faire alors de la mémoire compulsive qu'il qualifie de mémoire " ennemie », du fait de son omniprésence et de son rôle annihilateur? Que faire des souvenirs qui ressortaient par fragments décousus, mais qu'il ne pouvait pas taire? C'est finalement, dans un moment de désespoir, qu'il reviendra à lui en n'écrivant pas 
sur lui, mais sur une petite fille juive errant par les bois et les villages. C'est en pratiquant la fiction qu'il pourra enfin authentifier le réel qui jusqu'alors, dans ses écrits fidèles et vrais, lui paraissait fictionnel, inventé et donc non authentique.

L'enfant du génocide qu'est Appelfeld a peur de trahir, de mentir, d'omettre un détail, de sous-estimer un fait important, de commettre le moindre écart, sa mémoire « est une masse, si l'on peut dire, où ce qui est important et ce qui ne l'est pas se confondent, elle exige un élément dynamique qui la fasse bouger, lui donne des ailes - et c'est généralement ce que fait l'imagination $»^{53}$. L'imagination, prisonnière de la mémoire compulsive liée à la vie d'Appelfeld durant le génocide, ne peut se libérer qu'à l'instant où elle devient mémoire pour une petite fille juive victime du génocide. Appelfeld, libéré de son appréhension, de la hantise des survivants adultes et de leurs témoignages, libère aussi l'imagination qui n'est autre que sa propre vie réellement vécue, portée par une petite fille juive imaginaire. En libérant l'imagination qui est la source de la création, il met fin à la mémoire compulsive. Il ne s'agit pas pour autant d'inventer des faits nouveaux ni des images nouvelles, mais de procéder à un nouvel agencement de ces faits et images afin de rendre "visible 'l'idée' de l'auteur " ${ }^{54}$, en d'autres mots, sa perception de l'événement. Il ne s'agit pas de livrer ces faits et ces images dans un ordre chronologique, «d'empiler un fait sur un autre, mais de retenir les plus nécessaires, ceux qui abordent le cœur de l'expérience et non ses marges ${ }^{55}$. Appelfeld ne veut pas être l'auteur des "marges» de la Shoah, mais un écrivain, un artiste qui dispose maintenant de tous les procédés dont il a besoin pour écrire la Shoah, qui participe de la naissance de la littérature de la Shoah.

Les personnages auxquels Appelfeld donne vie demeurent cependant des enfants et le langage qu'il leur fait tenir est celui des enfants de la Shoah. Cette langue se distingue de celle des adultes, elle a une "nouvelle forme $»^{56}$. Elle puise à la source de la contemplation, des émotions, des cellules du corps-mémoire. Pour rompre avec cette perception d'une Shoah où «tout en elle semble déréel, au point qu'on dirait qu'elle n'appartient déjà plus à notre génération, mais qu'elle fait partie de la mythologie » et la «ramener dans le monde des hommes", car rien en elle n'est mystérieux ni mystique, il est nécessaire de «faire parler les événements à travers l'individu et son langage, de sauver la souffrance de l'énormité du nombre, de l'anonymat effroyable, de restituer à la personne son prénom et son nom de famille, de redonner à la personne torturée sa forme humaine, qui lui fut arrachée $\aleph^{57}$. Cette nouvelle forme, dira Appelfeld, « fut découverte par les enfants $»^{58}$ survivants.

Lorsque l'adulte survivant témoigne, il narre fidèlement et chronologiquement ce qui fut, ce qui est arrivé dans le passé. L'enfant survivant, dans ses témoignages-fictions, narre ce qu'il va arriver : «La question que j'avais devant moi désormais, dit Appelfeld, n'était plus : qu'est-il arrivé ?, mais : que faut-il qu'il arrive ? et, pour tout artiste, c'est

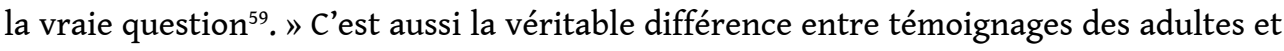
témoignages des enfants. Les enfants sont des témoins au futur. Danilo Kiš, dans son œuvre Sablier, avait instauré une telle distance ironique avec le génocide - l'événement passé - en faisant de cet événement un événement à venir, que ce procédé lui permettait de se transformer de témoin impossible en témoin possible, de témoin aveugle en témoin clairvoyant. Son ignorance due à son innocence en tant qu'enfant se transforme, avec la distance temporelle et l'écriture fictionnelle narrée au futur, en lucidité. L'événement à venir permet au père et au fils d'être égaux et ensemble face à l'événement à venir. Père et fils disparaitront ensemble dans l'événement à venir ${ }^{60}$. De 
même qu'Appelfeld imagine ce qui a déjà été vécu, Kiš imagine la disparition d'un père qui a déjà disparu. Pourtant tous deux imaginent, dans leur fiction du réel, que cela va arriver. Ce n'est qu'à cette condition qu'une écriture des enfants est possible. Cette écriture ne peut être que littéraire, puisqu'il s'agit de "dynamiser " par l'imagination cette masse informe qu'est la mémoire des enfants, c'est-à-dire lui donner une forme, un sens et les mots justes par la création artistique. C'est pourquoi, en effet, la littérature du génocide naît avec les enfants.

\section{Le janissaire}

Le génocide est " étrange ", son "étrangeté » réside en la négation de l'humain dans l'homme : c'est un humanicide. Qui plus que les Juifs étaient arrivés à ce degré de croyance en l'homme et sa liberté. Lorsqu'Appelfeld parle d'assimilation et de rejet de tout ce qui représentait la judéité, ne parle-t-il pas de cette foi en l'homme qui avait supplanté toute autre foi. Le communisme, auquel avaient adhéré des membres de sa famille, à sa façon convergeait vers la croyance en l'homme nouveau - sans foi. Or, le génocide ramène le Juif à sa judéité et le prive de son humanité. Par cette coupure radicale d'avec les hommes, d'avec la culture, c'est un retour forcé à la nature, à l'animalité, à l'état de bête. Dans ce processus inversé de l'initiation, leur âme a été raptée. Pour les enfants cachés comme Appelfeld, ce retour à la nature est réel (dans la forêt, parmi les bêtes). Ces enfants doivent désapprendre à être des humains, alors qu'ils naissent à la vie, pour apprendre à être des animaux. Pour qualifier cet état, $\mathrm{N}$. Zajde ${ }^{61}$ emploie le terme de désaffiliation: la perte de son monde, de sa famille, de sa culture, de sa religion, de sa langue. Ils deviennent étrangers à eux-mêmes et aux autres. Plus encore, ils deviennent des janissaires ${ }^{62}$.

Si l'adulte mesure la perte, l'enfant n'a rien qui puisse servir de mesure. Il sait une chose cependant : il a éprouvé le bonheur dans sa prime enfance et cette sensation-là, il ne la retrouve nulle part ailleurs. Ailleurs tout est hostile, horrible, dangereux, partout règne la peur. Dans ce retour à la nature, l'animal devient le semblable. Le chien est celui qui nous comprend et ne nous trahit pas, alors que l'homme devient un loup pour l'homme. Appelfeld parle de violence, de rage qui monte en lui et qui lui fait peur. Par là, nous mesurons l'impact sur les enfants de ce retour à la nature.

Le choix d'un narrateur-fille dans Tsili, plus précisément d'une « petite fille errant dans les bois » et non d'un " petit garçon ", n'est pas fortuit, d'abord pour instaurer une plus grande distance avec Appelfeld lui-même, nécessaire à l'écriture fictionnelle, mais aussi pour la différence fondamentale entre l'initiation féminine et l'initiation masculine.

$\mathrm{Au}$ contraire des femmes, nous dit M. Eliade, les hommes sont forcés, pendant leur période d'entraînement initiatique, de prendre conscience des réalités « invisibles " et d'apprendre une histoire sacrée qui n'est pas « évidente ", c'est-à-dire, n'est pas donnée dans l'expérience immédiate. Un néophyte comprend le sens de la circoncision après avoir appris le mythe d'origine. Tout ce qui lui arrive pendant l'initiation est dû au fait que certains événements ont eu lieu dans les Temps mythiques, et que ces événements ont modifié radicalement la condition humaine. L'initiation constitue, pour les garçons, l'introduction dans un monde qui n'est plus «immédiat»: le monde de l'esprit et de la culture. Pour les jeunes filles, au contraire, l'initiation comporte une série de révélations concernant le sens secret d'un phénomène apparemment «naturel»: le signe visible de leur maturité sexuelle ${ }^{63}$. 
L'initiation inversée, le retour à la nature, empêche l'introduction dans le monde de l'esprit et de la culture. Seule l'initiation des jeunes filles, qui consiste en la révélation d'un phénomène naturel, leur maturité sexuelle, ne semble pas être contrariée. Dans son roman Tsili, Appelfeld imagine une petite fille qui apprendra le sens secret des transformations de son corps. Après la rencontre avec Marek qui s'est enfui d'un camp, elle portera un enfant qui ne verra le jour que sous la forme d'un fœetus mort.

Danilo Kiš, dans son roman Sablier, imagine que E.S., qui symbolise le père de Kiš disparu à Auschwitz, a des douleurs menstruelles ${ }^{64}$. Mais, contrairement à la femme qui pourra donner la vie tant qu'elle aura des douleurs menstruelles parce qu'elle porte en elle la semence de la vie, les êtres de la désappartenance, tel E.S., souffrent de façon passagère et répétée, non pas parce qu'ils portent en eux la vie, mais parce qu'ils portent en eux la mort, leur propre mort. Êtres de la mort, ils ne peuvent donc que procréer la mort. Tsili, bien que "petite fille ", représente le "petit garçon » qu'était Appelfeld et, par conséquent, ne peut porter que la semence de la mort. De plus, tous les enfants ont eu leur âme ravie, aussi portent-ils en eux la mort, car comment pourraient-ils donner la vie quand ils sont déjà morts. Ainsi le principe «naturel » de la procréation, dans ce retour forcé à la nature, est lui aussi contrarié puisqu'il ne peut donner naissance qu'à la mort. Avec le génocide, la procréation n'est plus un phénomène naturel, mais contrôlé. Aux «fontaines de mort», les camps d'extermination, répondent les "fontaines de vie», les Lebensborn ${ }^{65}$ et les rapts d'enfants que synthétise bien le slogan: "Par le glaive et par le berceau ». Seuls les enfants nés de SS et de femmes sélectionnées sont considérés comme le «bon sang ". Seuls ils peuvent vivre et contribuer à la création de la «race aryenne». Les Lebensborn, en Europe de l'Ouest, sont des «haras" où se pratique «l'élevage humain». Les SS y sont qualifiés de "taureaux d'élevage». Notons ici que le vocabulaire utilisé relève du registre de la reproduction animale, ce qui correspond à cette situation de l'initiation inversée, du retour à l'état d'animal. Quant aux enfants raptés des territoires de l'Est - les enfants sélectionnés, car considérés aptes à la germanisation -, Clarissa Henry, Marc Hillel et Léon Poliakov les désignent du nom de «janissaires du III $^{e}$ Reich». Cette procréation et ces sélections contrôlées devaient produire des êtres de sang supérieur destinés aux territoires de l'Est qui devaient être vidés du sang des «êtres inférieurs ». D'où l'on comprend que non seulement l'homme est privé de son humanité, mais il est aussi privé de sa reproduction, les seules sources de vie étant les Lebensborn ${ }^{66}$.

37 À la fin de la guerre, il faudra d'urgence procéder au retour des rescapés parmi les humains, en particulier les enfants qui sont l'avenir de l'humain. Pour cela, il faudra annuler ce qui a précédé, de même qu'avait été annulé leur premier état par le processus génocidaire de l'initiation inversée. Il leur faudra devenir des hommes nouveaux, sans passé, repartir de rien. Le génocide a fait d'eux des hommes de la coupure radicale. Avec leur retour parmi les hommes, ils vivent une nouvelle coupure les empêchant de penser l'événement génocidaire. Ces humains de la coupure, de la rupture des liens, continuent de vivre leur état de janissaires.

\section{Le roman de l'étrangéisation}

L'adulte survivant, en tant que témoin, ne voudra pas se départir du «devoir de mémoire", du factuel, même si les faits semblent incroyables et faux, pensant 
authentifier l'événement par la preuve de sa parole. Pour les enfants survivants, au caractère déréel du génocide s'ajoute la délégitimation de leur témoignage, car, selon l'adulte survivant, l'enfant ne se souvient pas. À l'enfant privé du témoignage factuel reviennent la fiction du réel et le rôle de l'écrivain qui devra trouver la forme et le mot «juste» pour habiller leur corps-mémoire. L'enfant survivant, cependant, n'invente rien; toutes les images sont là. L'imagination dont il va user n'est pas de l'ordre de l'invention, mais de l'agencement. Il s'agit d'ordonner, de structurer ce qui a été déstructuré, de rassembler les fragments, de relier ce qui avait été rompu. En d'autres mots, de rendre réel l'irréel et de faire être ce qui a été.

39 La réalité génocidaire est si irréelle qu'elle en paraît imaginaire. La réalité a été étrangéisée. Les faits, les événements, de par leur incroyable réalité, sont devenus fiction, une fiction du réel. Il suffit à l'écrivain de leur donner une forme artistique, les « imaginer » s'il le faut, c'est-à-dire utiliser les faits authentiques comme matériau brut et leur donner, grâce à l'imagination, une nouvelle forme, une forme littéraire, une faction-fiction (documentaire-imaginaire) selon Danilo Kiš. Aussi ces faits, ces événements réels, parce qu'ils paraissent irréels, deviennent-ils « littéraires ». Et parce qu'il sera devenu fiction, le réel apparaitra dans toute sa réalité étrangéisée. L'écrivain s'attachera donc à transposer ces phénomènes en littérature. C'est la fiction du réel ou la faction/fiction. C'est le roman de l'étrangéisation ${ }^{67}$.

Lorsqu'il s'agit de décrire la réalité, l'art, par nature, réclame toujours une certaine intensification, une dose d'exagération. Or ce n'est pas le cas pour l'Holocauste, tant tout ce qui s'y rapporte paraît déjà profondément irréel, comme s'il n'appartenait plus au vécu de notre génération, mais à la mythologie ${ }^{68}$.

Contrairement à l'adulte qui est le témoin au passé, c'est-à-dire qu'il narre des faits du passé et au passé, l'enfant devient le témoin au futur, car il ne peut que projeter l'événement dans une narration fictionnelle. L'événement ne peut prendre corps que dans la fiction et à travers elle, par conséquent ce qui a eu lieu ne peut avoir réellement lieu que dans la création de la fiction du réel, en d'autres mots, ne peut être que dans le futur de la fiction et parfois même, comme dans le cas de Kiš, il n'a pas eu encore lieu, mais est un événement à venir, donc narré au futur. Cette narration au futur caractérise le témoignage des enfants qui ne peuvent être que des témoins au futur. «Je n'ai pas l'impression d'écrire sur le passé. Le passé en lui-même est un très mauvais matériau pour la littérature ${ }^{69}$.»

41 Si les uns et les autres veulent authentifier l'événement génocidaire, ils demeurent impuissants quant à son sens. Les faits, comme l'expriment les survivants, ne sont pas intelligibles, plus encore, ils semblent faux et les témoins eux-mêmes, en quelque sorte, des falsificateurs. L'adulte, malgré son désespoir de ne pas pouvoir comprendre l'événement, ne s'éloigne pas et ne veut pas s'éloigner des faits, car il "a le camp " incurablement. Le témoignage des enfants, qui ne peut être qu'une fiction du réel, qu'un roman de l'étrangéisation, veut aller «au cœur de l'expérience génocidaire » en nous livrant son expérience, sa perception. Par là, l'enfant nous livre son «idée » de l'événement. Ainsi le sens qui est donné par les enfants n'est qu'un sens singulier, propre à la singularité de l'auteur et de son expérience, et littéraire puisque ce sens ne peut exister qu'à travers l'œuvre littéraire qui le fait naître, et non le sens de l'événement génocidaire. 

du sens de l'humain : « Comment rendre à l'individu l'humanité et l'honneur dont il fut privé $^{70}$ ?»

\section{BIBLIOGRAPHIE}

APPELFELD, Aharon, « L'Holocauste lorsqu'on est enfant », Le Nouvel Observateur n ${ }^{\circ} 2097 \mathrm{du} 13 \mathrm{au}$ 19 janvier 2005.

APPELFELD, Aharon, L'héritage nu, Paris, Éditions de L'Olivier, 2006.

APPELFELD, Aharon, Et la fureur ne s'est pas encore tue, Paris, Éditions de L'Olivier, 2009.

APPELFELD, Aharon, Le garçon qui voulait dormir, Paris, Éditions de L’Olivier, 2011.

COQUIO, Catherine et KALISKY, Aurélia, L'enfant et le génocide : témoignages sur l'enfance pendant la Shoah, Paris, Robert Laffont, 2007.

FRIEDMAN, Carl, Mon père couleur de nuit, Paris, Denoël, 2000.

HILLEL, Marc et HENRY, Clarissa, Au nom de la race, Paris, Fayard, 1975.

ITZHAKI, Masha, Aharon Appelfeld : le réel et l'imaginaire, Paris, L'Harmattan, 2011.

KALISKY, Aurélia, «Quand tremblent les pactes. Poétique(s) de l'enfance traquée » in Adolphe Nysenholc (éd.), L'Enfant terrible de la littérature. Autobiographies d'enfants cachés, Bruxelles, Didier Devillez/Institut d'Études du Judaïsme, 2011, p. 233-309.

ELIADE, Mircea, Initiation, rites, sociétés secrètes, Paris, Gallimard, 1976.

PEJOSKA, Frosa, « L'écriture comme cénotaphe » in Catherine Coquio (dir.), L'histoire trouée:

négation et témoignage, Nantes, L'Atalante, 2003.

PEJOSKA, Frosa, « Danilo Kiš, la disparition étrangéisée », L’Intranquille nº 6-7, Paris 2001.

PEJOSKA-BOUCHEREAU, Frosa, « Le janissariat ou Au nom de l'Empire, au nom de la Nation, au nom du Parti, au nom de la Race ! ", Cahiers balkaniques n 36-37, direction Frosa Pejoska-Bouchereau, 2007-2008, p. 137-179.

PEJOSKA-BOUCHEREAU, Frosa, « Le roman de l'étrangéisation », in Écritures évolutives, sous la direction de Pierre Marillaud et Robert Gauthier, édité par l'Université Toulouse II-Le Mirail, CALS et Centre pluridisciplinaire de sémiologie textuelle, 2010, p. 265-277.

ZAJDE, Nathalie, Les enfants cachés en France, Paris, Odile Jacob, 2012.

\section{NOTES}

1. «Pendant des années, je me suis retenu de toute expression conceptuelle, tant je me méfiais du langage pluriel et des généralisations. Philip (Roth) m'a redonné un peu confiance en l'expression conceptuelle. Il m'a fallu me répéter à moi-même que l'expression conceptuelle, elle 
aussi, appartenait au vocabulaire des expressions humaines, et que nous n'avons d'autre choix que de l'humaniser et de la perfectionner. C'est pourquoi j'ai envers Philip de la gratitude.» Aharon Appelfeld, L'héritage nu, Paris, Éditions de L'Olivier, 2006, p. 18.

2. Aharon Appelfeld, «L'Holocauste lorsqu'on est enfant ", Le Nouvel Observateur n $2097 \mathrm{du} 13$ au 19 janvier 2005, p. 78.

3. «On ne doit pas jouer avec les mots ou avec la forme, on doit se contenter de raconter les choses telles qu'elles se sont passées, aussi précisément que possible. Il est rigoureusement interdit d'introduire un quelconque élément créatif extérieur au souvenir." A. Appelfeld, «L'Holocauste lorsqu'on est enfant », art. cit. p. 78 ; Histoire d'une vie, Paris, Éditions de L'olivier, 2004, p. 222.

4. A. Appelfeld, «L'Holocauste lorsqu'on est enfant », art. cit. p. 78.

5. A. Appelfeld, Histoire d'une vie, op. cit., p. 232.

6. Ibid.

7. A. Appelfeld, « L'Holocauste lorsqu'on est enfant », art. cit. p. 78.

8. Ibid. p. 79.

9. A. Appelfeld, Et la fureur ne s'est pas encore tue, Paris, Éditions de L'Olivier, 2009.

10. A. Appelfeld, Histoire d'une vie, op. cit., p. 211.

11. A. Appelfeld, "L'Holocauste lorsqu'on est enfant", art.cit. p. 79. Nous retrouvons l'illustration romanesque dans A. Appelfeld, Le garçon qui voulait dormir, Paris, Éditions de L'Olivier, 2011.

12. A. Appelfeld, L'héritage nu, op. cit., p. 57. Cf. aussi « [...] il arrive que, dans une discussion, on entende quelqu'un vous mettre en garde : "Laisse la littérature à l'écart de tout cela, le terrain est miné. Laisse parler les chiffres, laisse parler les documents et les faits établis." Je n'ai aucun désir de minimiser cette assertion. Mais je souhaite faire observer que les chiffres et les faits furent les moyens mêmes, bien avérés, des assassins. L'homme comme numéro est une des horreurs de la déshumanisation. Ils ne demandèrent jamais à quiconque qui il était ou ce qu'il était, ils tatouèrent des chiffres sur le bras. Devrions-nous chercher à suivre ce chemin et parler de l'homme dans la langue des statistiques?» A. Appelfeld, ibid., p. 56.

13. Nathalie Zajde, Les enfants cachés en France, Paris, Odile Jacob, 2012, p. 132, 137 et 139.

14. A. Appelfeld, Histoire d'une vie, op. cit., p. 150.

15. A. Appelfeld, L'héritage nu, op. cit., p. 79.

16. A. Appelfeld, Histoire d'une vie, op. cit., p. 8.

17. Ibid. p. 52.

18. Concept qui naît en 1991, lors de la Première réunion des enfants cachés à New York. On recense aujourd'hui environ 20000 enfants cachés en France. Cf. les travaux de Nathalie Zajde.

19. A. Appelfeld, Histoire d'une vie, op. cit., p. 8-9 et L'héritage nu, op. cit., p. 33.

20. A. Appelfeld, Histoire d'une vie, op. cit., p. 71 et p. 74 .

21. Ibid. p. 62.

22. Ibid. p. 73-74.

23. Ibid. p. 161.

24. Ibid. Cf. également «Dans l'état de contemplation, on recule à l'intérieur de soi, on baigne dans une musique intérieure. On se construit un abri, ou parfois on s'élève pour observer de loin. ", ibid. p. 168.

25. Ibid. p. 163-165.

26. Ibid. p. 192.

27. Ibid.

28. Ibid. p. 166-167.

29. Ibid. p. 168.

30. Ibid. p. 9 et p. 109-113.

31. Ibid. p. 66. 
32. Ibid. p. 223.

33. Ibid.

34. Ibid. p. 200

35. Ibid. p. 203

36. Ibid.

37. Carl Friedman, Mon père couleur de nuit, Paris, Denoël, 2000.

38. Ibid. p. 31.

39. A. Appelfeld, Histoire d'une vie, op. cit., p. 214-215.

40. N. Zajde, Les enfants cachés en France, op. cit., p. 12-13.

41. Ibid.

42. «Les gens se remplirent de silence. Tout ce qui était arrivé était à tel point démesuré et inconcevable que le témoin même se voyait en falsificateur. Le sentiment que votre expérience ne peut être racontée, que personne ne peut la comprendre, est sans doute la pire impression que les survivants éprouvèrent après la guerre. Ajoutez-y le sentiment de culpabilité et vous verrez que vous aurez construit, de vos propres mains et pour vous-même, un vaste programme de malentendus. » A. Appelfeld, L'héritage nu, op. cit., p. 61-62.

43. Ibid. p. 11.

44. Ibid. p. 9-10.

45. Ibid. p. 11.

46. Ibid. p. 9-10.

47. A. Appelfeld, Histoire d'une vie, op. cit., p. 10.

48. A. Appelfeld, L'héritage nu, op. cit., p. 11.

49. Ibid.

50. Ibid. p. 12.

51. Danilo Kiš, auteur qui fut enfant pendant le génocide, reniera aussi son premier roman Psaume 44, car il y regrettera le manque de distance dans l'écriture qu'il s'évertuera par la suite de corriger, considérant qu'il a commis une faute majeure : «La faiblesse de ce livre de jeunesse ne réside pas tant dans cette intrigue, trop forte, trop pathétique, que dans l'absence fatale de la moindre distance ironique - élément qui deviendra par la suite partie intégrante de mon procédé littéraire. » Ce manque de distance est aussi perçu par le lecteur puisque les membres du jury, parmi lesquels se trouvaient un poète surréaliste et un romancier, furent persuadés, avant de découvrir le nom de l'auteur, que le roman avait été écrit par une femme, non seulement à cause d'une certaine "psychologie féminine ", mais aussi à cause de la "sensibilité féminine " qu'ils trouvèrent à ce texte ! Cf. notre texte "L'écriture comme cénotaphe ", in Catherine Coquio (dir.) L'histoire trouée : négation et témoignage, Nantes, L'Atalante, 2003.

52. A. Appelfeld, L'héritage nu, op. cit., p. 12.

53. Ibid. p. 14.

54. Ibid.

55. Ibid.

56. Ibid. p. 67.

57. Ibid. p. 72-73.

58. Ibid. p. 67.

59. Ibid. p. 13.

60. Cf. notre texte : « Danilo Kiš, la disparition étrangéisée ", L'Intranquille n ${ }^{\circ}$ 6-7, Paris 2001.

61. N. Zajde, Les enfants cachés en France, op. cit., p. 205.

62. Ibid. p. 27, p. 33 et p. 119.

63. Mircea Eliade, Initiation, rites, sociétés secrètes, Paris, Gallimard, 1976, p. 108.

64. «Je l'avoue carrément : mon cœur a des menstruations. Tardives, douloureuses règles de ma judéité... Le monsieur que vous voyez passer, honorables Dames et Messieurs, ce monsieur de cinquante ans environ, en costume gris, avec des lunettes à monture d'acier, une canne et une 
étoile jaune (que vous ne voyez pourtant pas car il la cache derrière son porte-documents), ce monsieur, eh bien, il a des règles. Messieurs les juges, mon cœur a des règles. Déviation biologique, incarnation du principe juif, féminin. Nouvelle à sensation pour les journaux : un monsieur grisonnant à des douleurs menstruelles! Le plus intéressant, c'est qu'il s'agit d'un homme en parfaite santé physique (en excluant un léger rhume), d'un homme chez qui l'on n'a pas noté le moindre trouble des fonctions glandulaires ou hormonales. Règles masculines? Non. Principe féminin porté à ses conséquences extrêmes. Fleur menstruelle du cœur. Semence de la mort. Weltschmertz. [...] Pour ce qui est de cette lettre (Madame), le monsieur qui vous l'a écrite (je sais, cela sonne bizarrement), ce monsieur va accoucher! Son analyse d'urine le montre clairement. Comme elle montre tout aussi clairement qu'il s'agit d'un homme. C'est tout. Puisque vous avez dit que c'est votre frère, conseillez-lui de se préparer. Il va concevoir, Madame. Il porte en lui la semence de la mort. Condoléances, chère Madame. » Cf. notre texte : "Danilo Kiš, la disparition étrangéisée ", op. cit.

65. Créé le 31 décembre 1931 sur ordre d'Heinrich Himmler, le RuSHA (l'Office supérieur de la Race et du Peuplement), dont l'un des premiers travaux est d'établir un tableau officiel des "valeurs raciales", met au point une série de remèdes nationaux-socialistes, parmi lesquels figure l'élevage des enfants dans des maisons d'État, qui annoncent les "Fontaines de vie " (Lebensborn). Dans la perspective de « l'hygiène de la race et de son contrôle dans l'intérêt de la patrie ", selon les travaux du docteur Wilhelm Schallmayer, il était procédé à un contrôle eugénique permanent de la population allemande. Pour ce théoricien de la race aryenne, l'arrêt de la reproduction des éléments non valables était aussi important que la reproduction des éléments valables. Ce qui explique la coexistence d'une politique de «stérilisation» et de «castration» et d'une politique d'élevage sélectionné. La sélection du mâle en mesure de reproduire des éléments valables était assurée par les SS. Celle des femmes sera prise en charge par la "Société enregistrée Lebensborn", créée le 12 décembre 1935 à l'initiative du RuSHA. L'Organisation Lebensborn est placée sous la direction personnelle de Himmler, elle fait partie intégrante du Bureau central de la SS pour la Race et le Peuplement. Dans une circulaire du 13 septembre 1936, Himmler fixe les tâches de cette organisation. Elle précise que tout Führer SS devra avoir au moins quatre enfants de bonne valeur raciale. S'il n'a pas d'enfants, il devra en adopter. L'organisation devra aider les chefs de la SS dans la sélection et l'adoption d'enfants qualifiés. Cf. Marc Hillel et Clarissa Henry, Au nom de la race, Paris, Fayard, 1975.

66. Cf. notre étude : « Le janissariat ou Au nom de l'Empire, au nom de la Nation, au nom du Parti, au nom de la Race!», Cahiers balkaniques $n^{\circ} 36-37$, direction Frosa Pejoska-Bouchereau, 2007-2008, p. 137-179.

67. Cf. notre travail : "Le roman de l'étrangéisation ", in Écritures évolutives, sous la direction de Pierre Marillaud et Robert Gauthier, édité par l'Université Toulouse II-Le Mirail, CALS et Centre pluridisciplinaire de sémiologie textuelle, 2010, p. 265-277. Sur l'autobiographie, le témoignage et la fiction, nous renvoyons au travail d'Aurélia Kalisky : "Quand tremblent les pactes. Poétique(s) de l'enfance traquée », in Adolphe Nysenholc (éd.), L'Enfant terrible de la littérature. Autobiographies d'enfants cachés, Bruxelles, Didier Devillez / Institut d'Études du Judaïsme, 2011, p. 233-309.

68. A. Appelfeld, « L'Holocauste lorsqu'on est enfant », art. cit., p. 79.

69. A. Appelfeld, Histoire d'une vie, op. cit., p. 151.

70. A. Appelfeld, L'héritage nu, op. cit., p. 16. 


\section{RÉSUMÉS}

Aharon Appelfeld établit une distinction entre les témoignages des adultes sur le génocide et ceux des enfants. Si pour les adultes les témoignages doivent être factuels, fidèles et chronologiques, pour les enfants, que l'on a refusé de considérer comme des témoins, faute de pouvoir reconstituer le passé par la mémoire, il s'agit de recourir à l'invention, à l'expression des sensations et des sentiments, en d'autres mots à la perception, pour procéder à une « reconstruction». Ce recours singulier à l'imaginaire et aux sens serait, selon Appelfeld, à l'origine de la naissance de la littérature de la Shoah.

Notre questionnement aura pour sujet la définition du «témoignage » et sa place dans la littérature.

«Les enfants survivants ne sauraient se remémorer l'Holocauste à la manière des adultes. Leur contribution est inséparable de leur expérience vécue. Mais cette expérience, quoique limitée, est profonde. Rien d'étonnant à ce que la littérature de l'Holocauste soit née avec eux. » (Aharon Appelfeld, «L'Holocauste lorsqu'on est enfant», Le Nouvel Observateur n ${ }^{\circ} 2097 \mathrm{du} 13 \mathrm{au}$ 19 janvier 2005).

Aharon Appelfeld differentiates between the adult testimonies on genocide and those of children. Whereas in the case of adults the testimonies have to be factual, reliable and chronological, in the case of children-who are refused the status of witnesses because of their incapacity to reconstruct the past from memory-the "reconstruction" is based on invention, sensory elements and feelings, in other words on perception. According to Appelfeld, this particular resort to imagination and senses is at the origins of the Holocaust literature.

My intervention will focus on the definition of the "testimony" and on its place within the literary work.

"Surviving children could not rememorize the Holocaust in the same way as adults. Their contribution is inseparable from the experience they lived. Yet, despite being limited, this experience is profound. It is therefore not astonishing that the Holocaust literature should be born with them." (Aharon Appelfeld, "Holocaust through the eyes of the children", Le Nouvel Observateur 2097, 13-19 January 2005).

אפלפלד מבחין בין עדויות בוגרים על השואה ובין אלו של ילדים. בעוד שעדויות הבוגרים הן אמינות ונשענות על עובדות, אלו של הילדים אינן ממש עדויות בשל הקושי של הילדים לשחזר את העודי שעלר.

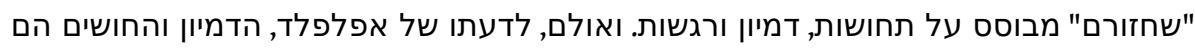

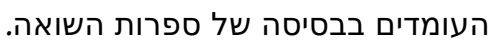
מאמרי יעסוק בהגדרת העדות ומקומה ביצירה הספרות השואה ספית. 
INDEX

Thèmes : littérature

מילות מפתח

שואה, עדים, עדי העתיד, ניצולים, ילדים במחבוא, כתיבה מתעדת, זיכרון, רומן ההזרה,:

לבנסבורן

Mots-clés : génocide, Holocauste, témoins, témoins au futur, survivants, enfants, enfants cachés, écriture testimoniale, mémoire, roman de l'étrangéisation, Lebensborn, janissaire

Keywords : witness, testimony, memory, hidden children, Lebensborn, Janissary, literature, Holocaust

Index chronologique : Shoah

\section{AUTEUR}

FROSA PEJOSKA-BOUCHEREAU

INALCO 